\title{
Evaluación de la confiabilidad y la estructura factorial de tres escalas de procrastinación crónica
}

\author{
Doris Argumedo Bustinza ${ }^{1}$, Karem Díaz Cerna ${ }^{2}$ y Arturo Calderón García ${ }^{3}$ \\ Pontificia Universidad Católica del Perú
}

\author{
Juan Francisco Díaz-Morales ${ }^{4}$ \\ Universidad Complutense de Madrid
}

Joseph R. Ferrari ${ }^{5}$

Universidad DePaul

\begin{abstract}
Este estudio explora la confiabilidad y estructura factorial de tres escalas que evalúan la procrastinación crónica: Escala de Procrastinación General (EPG, Lay, 1986), Inventario de Procrastinación para Adultos (IPA, McCown \& Johnson como se cita en Ferrari, Johnson \& McCown, 1995) y Escala de Procrastinación en la Toma de Decisiones (PTD, Mann, 1982). La muestra estuvo compuesta por 514 adultos entre 20 y 65 años de Lima Metropolitana. Las tres escalas mostraron altos niveles de consistencia interna y los análisis factoriales indicaron una solución de tres factores para EPG e IPA y un solo factor para PTD. Un análisis factorial de segundo orden sugirió la existencia de un sólo factor a la base de las agrupaciones de los ítemes de las escalas EPG e IPA. No se observaron diferencias teóricamente relevantes en la procrastinación crónica según sexo, edad y nivel de instrucción, sin embargo, el estrato socio-económico indicó mayores niveles de procrastinación crónica en el sector más pobre.
\end{abstract}

Palabras clave: procrastinación crónica, análisis factorial, confiabilidad, adultos peruanos.

Assessment of the confiability and factorial structure of three scales measuring chronic procrastination

This study explores the confiability and factorial structure of three scales measuring chronic procrastination: Scale of General Procrastination (EPG, Lay, 1986), Adult Procrastinatuon Inventory (IPA, McCown \& Johnson as cited in Ferrari, Johnson \& McCown, 1995) and the Scale of Procrastination in Decision-Making (PTF, Mann, 1982). The sample included 514 adults between 20 and 65 years of age from Lima. The three scales showed high levels of internal consistency and factorial analysis showed three factors for EPG and IPA and one factor for PTD A second degree factorial analysis suggested the presence of only one factor based on the grouping of items of the EPG and IPA scales The study did not find theoretically relevant differences in chronic procrastination according to gender, age or education level. However, with respect to socioeconomic status, there were higher levels of chronic procrastınation in the poorest sector

Key words: chronic procrastınation, factorial analysıs, confiability, peruvian adults

1 Licenciada en Psicología Clínica Profesora auxiliar y coordinadora de la Especialidad de Psicología. Especialista en psicometría y metodología de investigación. Correo electrónico: dargume@ @ucp.edu.pe 

Procrastination en el idioma inglés es un término que describe la postergación para realizar actividades (Merriam-Webster, 2004). En ambientes clínicos, el estudio de las conductas de postergación desde 1977 ha dado lugar a que se diferencien conductas de postergación y de procrastination (Ellis \& Knaus, 1977). Las primeras refieren el común aplazamiento de una tarea con el fin de darle prioridad a otra más productiva en ese momento y que no constituye un perjuicio para la persona. Las segundas hacen referencia más bien a un desorden clínico caracterizado por la intención de hacer una tarea y una falta de diligencia ya sea para empezarla, para desarrollarla o para finalizarla, cuyo proceso generalmente se acompaña de sentimientos de nerviosismo o inquietud y abatimiento (Ferrari, Johnson \& McCown, 1995; Steel, 2003).

Procrastination no alude entonces necesariamente a la falta de finalización de la tarea, sino a la experiencia de la postergación que tiene como consecuencia en algunos casos no terminarla. Así, generalmente se termina aunque tarde. Para diversos autores (Lay, Schouwenburg, Ferrari, Pychyl, comunicación personal, 6 Agosto, 2003) es esta la característica que ha permitido que no se le preste

2 Licenciada en Psicología Clínica. Docente del Departamento de Psicología. Su línea de investigación comprende estudios de personalidad y procrastinación. Correo electrónico: kdiaz@pucp.edu.pe

3 Ingeniero Estadístico e Informático. Profesor de la sección Matemáticas. Especialista en métodos cuantitativos aplicados a las Ciencias Sociales y del Comportamiento. Correo electrónico: acalder@pucp.edu.pe

+ Doctor en Psicología, Profesor Ayudante. Correo electrónico: juanfcodiaz@ psi.ucm.es

5 Doctor en Psicología. Profesor de Psicología. Correo electrónico: jferrari@ depaul. edu 
considerable atención en la investigación clínica. Sus consecuencias sólo se observan en el largo plazo. Ferrari (1992a, 2001) señala que éstas incluyen un desempeño más pobre en relación con sus pares y colegas en el ámbito personal, social o profesional y menor creatividad en sus proyectos.

Los tipos de procrastination son dos. Procrastinación Situacional (Situational Procrastination), describe el hábito de procrastinar solamente en determinados contextos, por ejemplo, el académico. El segundo tipo es llamado Procrastinación Crónica (Chronic Procrastination) y hace referencia a un patrón no adaptativo para la vida del individuo que se traduce en una postergación que trasciende a todas las situaciones y contextos (Ferrari et al., 1995; Lay \& Schouwenburg, 1993).

Schouwenburg, Lay, Pychyl y Ferrari (2004) señalan que la forma crónica ha dado lugar al estudio de diversas variables asociadas y se han identificado determinados rasgos de personalidad asociados a este trastorno, sobretodo aquellos referidos a la organización, planificación y autorregulación para la búsqueda y obtención de metas.

En países hispanohablantes no se encuentra un término que pueda utilizarse para designar hábitos de postergación disruptivos y desadaptativos. El diccionario de la Real Academia Española nombra y define el término procrastinación como una falta de diligencia en la realización de la tarea y nombra como sus sinónimos dilatar y aplazar, aunque no se incluye a su vez procrastinación en las definiciones de estas palabras (Real Academia Española, 2002).

Si se asocia a las características de la población de habla hispana, sorprende la falta de una palabra que describa aquellas conductas que denoten la intención de hacer una tarea y su aplazamiento 
así como las emociones negativas usualmente presentes. Si bien el término procrastinación involucra la mayor parte de estas características y su uso clínico obliga a incorporar en la definición la experiencia subjetiva descrita, no es un término reconocido, utilizado o incluso nombrado en algún otro diccionario en español.

La diferenciación entre hábitos sociales de demora y postergación en el contexto peruano y el hábito de procrastinar no es clara. En el ámbito personal, es necesario hacer un deslinde entre costumbres sociales y un patrón recurrente que causa una mala adaptación del individuo a su medio, es decir, estudiar si es posible hablar de personas que presenten un desorden de procrastinación y observar sus características y rasgos asociados. Para ello, es imprescindible tener los instrumentos necesarios para poder evaluar este fenómeno, estudiar su manifestación en nuestra población y finalmente, establecer si, a pesar de la costumbre social, ocurre la procrastinación descrita en los ambientes clínicos (Hernández, Fernández \& Baptista, 2000).

Este artículo se centra en el estudio y validación de instrumentos que permitan identificar la procrastinación en su forma crónica. Para ello, se analiza la confiabilidad y la estructura factorial de tres instrumentos psicométricos diseñados para evaluar procrastinación en población angloamericana: la Escala de Procrastinación General (EPG, Lay, 1986), el Inventario de Procrastinación para Adultos (IPA, McCown \& Johnson como se cita en Ferrari et al., 1995) y el Cuestionario de Procrastinación en la Toma de Decisiones (PTD, Mann, 1982).

La adaptación de tests psicológicos a las particularidades de una cultura constituye un proceso complejo que no solo implica la traducción idiomática del mismo sino la valoración de la pertinencia del constructo psicológico en el nuevo contexto cultural para luego comprobar su equivalencia dentro del marco conceptual que 
lo sustenta y con los tests ya existentes. Este es un tema central en la nueva tendencia de estudios interculturales, así en la actualidad resulta de vital importancia la comparación de las propiedades psicométricas de los instrumentos propios de la población en la que fueron diseñados con los hallados en un nuevo contexto cultural antes de desarrollar cualquier estudio transcultural.

\section{Metodología}

\section{Participantes}

La muestra total de participantes quedó conformada por 514 adultos entre 20 y 65 años de edad $(M=38.79, D S=12.08)$, distribuidos homogéneamente según sexo $(50.78 \%$ de mujeres y $49.22 \%$ de varones) y provenientes de los cinco niveles socioeconómicos correspondientes a los estratos $\mathrm{A}, \mathrm{B}, \mathrm{C}, \mathrm{D}$ y $\mathrm{E}$ que conforman el universo de población limeña (Apoyo, Opinión y Mercado, 2002).

Con el objetivo de mantener la representatividad de la muestra de la población limeña se buscó que el 50\% (260) estuviese constituido por sujetos pertenecientes al sector D-E, el 28\% (144) corresponda a participantes del sector C, el $18 \%$ (90) de la muestra esté constituida por el sector B y el $3.89 \%$ (20) de participantes representen al sector A. En relación con el grado de instrucción, el 59.14\% (304) de los participantes contaban con estudios secundarios, seguido de un $20 \%$ (103) que reportó estudios de instrucción técnica y un $20.80 \%$ (107) que señaló tener una educación superior universitaria y/o estudios de post grado universitario (Cuadro 1). 
Evaluación de la confiabilidad y la estructura factorial de tres escalas

\section{Cuadro 1}

Descripción de la muestra

\begin{tabular}{llcc}
\hline & & $N$ & $\%$ \\
\hline Edad & $20-40$ & 262 & 51.0 \\
& $41-65$ & 252 & 49.0 \\
\hline \multirow{2}{*}{ Sexo } & Mujer & 261 & 50.8 \\
& Varón & 253 & 49.2 \\
\hline \multirow{2}{*}{ Instrucción } & Secundaria & 304 & 59.1 \\
& Superior técnica & 103 & 20.0 \\
& Universitaria/Post grado & 107 & 20.8 \\
\hline \multirow{2}{*}{ Nivel Socioeconómico } & A & 20 & 3.9 \\
& B & 90 & 17.5 \\
& C & 144 & 28.0 \\
& D-E & 260 & 50.6 \\
\hline Total & & 514 & \\
\hline
\end{tabular}

\section{Instrumentos}

Escala de Procrastinación General (Lay, 1986)

Es una escala unidimensional compuesta por 20 ítemes (escala tipo Lickert de 5 puntos). Diez ítemes tienen puntaje reverso y se suman las respuestas para obtener un puntaje global. Incluye ítemes como "Preparo mi ropa la noche anterior a una entrevista para que no se me haga tarde", "Me doy cuenta que suelo correr contra el reloj", "En el último año, el dejar las cosas para último minuto me ha perjudicado en términos económicos y/o de oportunidades". Los puntajes altos obtenidos en la prueba reflejan una conducta procrastinadora que se asocia a una necesidad de activación fisiológica para desempeñar la tarea (Ferrari,1992b). En investigaciones previas la escala ha presentado un Alfa de Cronbach de .82 (Lay, 1986), .78 (Ferrari, 1991) y una confiabilidad test-retest ( 1 mes) de .80 . 
Inventario de Procrastinación para Adultos (McCown \& Johnson como se cita en Ferrari et al., 1995)

Es una escala unidimensional de 15 ítemes en una escala tipo Lickert de 5 puntos con siete ítemes con puntaje reverso. Se obtiene un único puntaje de procrastinación sumando las respuestas de todos los reactivos. Un puntaje alto en esta escala indica frecuentes demoras en realizar tareas como una forma de evitar poner en cuestionamiento las capacidades de la persona (Ferrari et al., 1995). Presenta ítemes como "Frecuentemente me doy cuenta que estoy haciendo tareas que me había propuesto hacer con anterioridad", "Me toma días realizar trabajos, incluso los que sólo requieren sentarse y hacerlos", "Cuando estoy haciendo un trabajo que debo presentar, con frecuencia pierdo tiempo haciendo otras cosas". Los autores reportaron un coeficiente Alfa de .79 y confiabilidad test-retest (un mes) de .71. Otras investigaciones reportan un coeficiente Alfa de .86 y test-retest (6 meses) de .76. (Ferrari, 2000).

Cuestionario de Procrastinación en la Toma de Decisiones (Mann, 1982)

Examina el aspecto cognitivo de la procrastinación frente a situaciones importantes de toma de decisiones. La escala se basa en la teoría del conflicto para tomar decisiones (Ferrari \& Dovidio, 2000), la cual considera la procrastinación (es decir, indecisión) como un patrón desadaptativo utilizado por las personas cuando se enfrentan a conflictos y deben escoger alternativas. Está compuesta de 5 ítemes en una escala de 5 puntos de tipo Lickert. El autor reportó un Alfa de Cronbach de .72 y .80 para el instrumento y un test-retest ( 1 mes) de .62 y .69 .

\section{Procedimiento}

La estrategia de recolección de datos supuso un muestreo por cuotas (Hernández et al., 1998), lo cual implicó la inclusión de sujetos según algunos criterios específicos que aseguraran la representatividad 
de la muestra por sexo, grupo de edad y estrato socioeconómico. Un total de ocho entrevistadores aplicaron los tres instrumentos de procrastinación que fueron adaptados al español siguiendo los procedimientos formales de doble traducción (Díaz, 2004).

Luego se desarrollaron los análisis de confiabilidad, discriminación de ítemes y estructura factorial para cada escala. Finalmente se desarrollaron los análisis pertinentes para determinar la existencia de diferencias significativas en el nivel de procrastinación medido a través de las tres escalas según las diversas variables socio-demográficas de interés.

\section{Resultados}

\section{Discriminación de los útemes y confiabilidad de las escalas}

Con respecto a la Escala de Procrastinación General, el análisis de las correlaciones ítem-test mostró que el ítem 5 ("usualmente respondo e-mails varios días después de haberlos recibido") no correlacionó con la escala $(r=0.07)$, así como tampoco el ítem 11 ("cuando me preparo para salir, rara vez tengo que hacer algo a último minuto") $(r=0.00)$, por lo tanto se decidió su neutralización. Este procedimiento se refiere a otorgar el puntaje promedio de la escala (en este caso el valor de 3) a todos los sujetos del estudio en dichos reactivos. Los nuevos coeficientes de correlación ítem-escala total se ubicaron entre 0.27 y 0.58 y el coeficiente Alfa de Cronbach fue de 0.81 .

Para el Inventario de Procrastinación para Adultos, las correlaciones ítem-test estuvieron en un rango de 0.13 a 0.55 . El ítem 6 ("si se dictase un curso sobre cómo hacer las cosas a tiempo, yo iría") presentó el coeficiente de correlación más bajo $(r=0.13)$ con la escala, por lo tanto se siguió el procedimiento ya mencionado de neutralización para este ítem. En consecuencia, las nuevas correlaciones ítem-test oscilaron entre 0.25 y 0.56 y el coeficiente Alfa de Cronbach para el inventario fue de 0.81 . 
El Cuestionario de Procrastinación en la Toma de Decisiones mostró correlaciones ítem-escala total entre 0.56 y 0.68 . El coeficiente Alfa de Cronbach para esta escala fue también adecuado $(\alpha=0.84)$.

Se observa que cada una de las tres escalas de procrastinación presentan índices adecuados $(\alpha \geq 0.80)$ dado que suponen un alto nivel de consistencia al interior de las mismas (Cuadro 2). Estos resultados son consistentes con los reportados en investigaciones en otros contextos culturales, así como con lo encontrado con una muestra de estudiantes universitarios limeños (Díaz, 2004; Ferrari et al., 1995).

\section{Cuadro 2}

Confiabilidad y discriminación ítem-test para cada escala de procrastinación crónica

\begin{tabular}{cccccc}
\hline $\begin{array}{c}\text { Escala de } \\
\text { Procrastinación } \\
\text { General }\end{array}$ & $\begin{array}{c}\text { Inventario de } \\
\text { Procrastinación para } \\
\text { Adultos }\end{array}$ & $\begin{array}{c}\text { Cuestionario de } \\
\text { Procrastinación en la } \\
\text { Toma de Decisiones }\end{array}$ \\
\hline Item & Discriminación & Item & Discriminación & Item & Discriminación \\
\hline EP1 & 0.27 & IA1 & 0.48 & PD1 & 0.56 \\
EP2 & 0.38 & IA2 & 0.57 & PD2 & 0.62 \\
EP3 & 0.33 & IA3 & 0.44 & PD3 & 0.56 \\
EP4 & 0.29 & IA4 & 0.46 & PD4 & 0.68 \\
EP6 & 0.30 & IA5 & 0.48 & PD5 & 0.60 \\
EP7 & 0.42 & IA7 & 0.54 & & \\
EP8 & 0.38 & IA8 & 0.37 & & \\
EP9 & 0.48 & IA9 & 0.39 & & \\
EP10 & 0.31 & IA10 & 0.31 & & \\
EP12 & 0.50 & IA11 & 0.25 & & \\
EP13 & 0.33 & IA12 & 0.53 & & \\
EP14 & 0.34 & IA13 & 0.37 & & \\
EP15 & 0.47 & IA14 & 0.49 & & \\
EP16 & 0.42 & IA15 & 0.44 & & \\
EP17 & 0.47 & & & & \\
EP18 & 0.46 & & & & \\
EP19 & 0.58 & & & & \\
EP20 & 0.36 & & & & \\
\hline Alfa & 0.81 & & & & \\
\hline
\end{tabular}


Evaluación de la confiabilidad y la estructura factorial de tres escalas

\section{Estructura factorial de las escalas}

Con el fin de establecer la estructura interna de cada una de las escalas, se condujo un análisis factorial de componentes principales con rotación Oblimin. El análisis de la Escala de Procrastinación General de Lay (1986) señaló una solución factorial de tres factores con valores propios mayores que $1(4.42,2.18$ y 1.19$)$ (método de Kaiser), encontrándose que estos factores explican el $43.27 \%$ de la varianza total $(24.58,12.09$ y 6.60 respectivamente para cada factor) (Cuadro 3). La adecuación de la muestra fue confirmada a través del índice Kaiser-Mayer-Olkin $(\mathrm{KMO}=0.86)$.

\section{Cuadro 3}

Escala de Procrastinación General: correlaciones item-factor y comunalidades con rotación Oblimin

\begin{tabular}{lcccc}
\hline & \multicolumn{3}{c}{ Correlaciones ítem-factor } & \\
\cline { 2 - 4 } Item & 1 & 2 & 3 & Comunalidad \\
\hline EP1 & 0.28 & -0.48 & -0.26 & 0.34 \\
EP2 & 0.26 & -0.61 & -0.01 & 0.38 \\
EP3 & 0.27 & -0.12 & 0.65 & 0.45 \\
EP4 & 0.46 & -0.01 & 0.27 & 0.25 \\
EP6 & 0.30 & -0.02 & 0.64 & 0.43 \\
EP7 & 0.48 & -0.50 & -0.16 & 0.43 \\
EP8 & 0.50 & -0.12 & 0.31 & 0.28 \\
EP9 & 0.61 & -0.44 & -0.06 & 0.48 \\
EP10 & 0.14 & -0.63 & -0.05 & 0.40 \\
EP12 & 0.58 & -0.50 & -0.05 & 0.48 \\
EP13 & 0.31 & -0.09 & 0.62 & 0.42 \\
EP14 & 0.33 & -0.15 & 0.51 & 0.32 \\
EP15 & 0.69 & -0.11 & 0.30 & 0.49 \\
EP16 & 0.19 & -0.75 & 0.09 & 0.58 \\
EP17 & 0.21 & -0.77 & 0.20 & 0.66 \\
EP18 & 0.68 & -0.07 & 0.33 & 0.51 \\
EP19 & 0.63 & -0.48 & 0.16 & 0.49 \\
EP20 & 0.55 & 0.02 & 0.39 & 0.39 \\
\hline Correlaciones entre factores & & \\
Factor 1 & & & & \\
Factor 2 & -0.30 & 1.00 & & \\
Factor 3 & 0.26 & 0.06 & & \\
\hline
\end{tabular}


Un análisis teórico de cada factor reveló que no existen diferencias conceptuales claramente establecidas entre cada uno de ellos en relación con la procrastinación crónica. Es decir, no es posible establecer áreas diferenciadas que, en su conjunto, permitan comprender el constructo de procrastinación crónica. Se condujo entonces un análisis factorial de segundo orden para analizar si la agrupación de factores responde a una sola variable a la base. Los resultados muestran una solución unifactorial con un valor propio de 1.72 , que explica el $57.39 \%$ de la varianza total (Cuadro 4).

\section{Cuadro 4}

Valores propios, porcentajes de varianza y porcentajes acumulados en el análisis factorial de segundo orden de la Escala de Procrastinación General

\begin{tabular}{cccc}
\hline Componente & Valor propio & \% de varianza & \% acumulado \\
\hline 1 & 1.72 & 57.39 & 57.39 \\
2 & 0.84 & 27.86 & 85.25 \\
3 & 0.44 & 14.75 & 100.00 \\
\hline
\end{tabular}

Con respecto al Inventario de Procrastinación para Adultos de McCown y Johnson (como se cita en Ferrari et al., 1995) se encontraron tres factores con valores propios mayores que 1 (4.20, 21.59 y 1.00 ) (método de Kaiser), que explicaban el $48.60 \%$ de la varianza total $(30.02,11.37$ y 7.20 respectivamente para cada factor) (Cuadro 5). La adecuación de la muestra fue confirmada a través del índice Kaiser-Mayer-Olkin $(K M O=0.87)$. 
Evaluación de la confiabilidad y la estructura factorial de tres escalas

\section{Cuadro 5}

Inventario de Procrastinación para Adultos: correlaciones ítem-factor y comunalidades con rotación Oblimin

\begin{tabular}{ccccc}
\hline & \multicolumn{3}{c}{ Correlaciones item-factor } & \\
\cline { 2 - 4 } Item & 1 & 2 & 3 & Comunalidad \\
\hline IA1 & 0.60 & 0.40 & 0.21 & 0.42 \\
IA2 & 0.79 & 0.36 & 0.21 & 0.65 \\
IA3 & 0.36 & 0.65 & 0.21 & 0.47 \\
IA4 & 0.63 & 0.03 & 0.45 & 0.48 \\
IA5 & 0.34 & 0.24 & 0.67 & 0.48 \\
IA7 & 0.50 & 0.19 & 0.64 & 0.49 \\
IA8 & 0.38 & 0.57 & 0.09 & 0.38 \\
IA9 & 0.18 & 0.18 & 0.71 & 0.53 \\
IA10 & 0.18 & 0.00 & 0.65 & 0.43 \\
IA11 & 0.04 & 0.72 & 0.12 & 0.56 \\
IA12 & 0.69 & 0.47 & 0.16 & 0.56 \\
IA13 & 0.31 & 0.64 & 0.13 & 0.43 \\
IA14 & 0.64 & 0.04 & 0.49 & 0.51 \\
IA15 & 0.48 & 0.05 & 0.55 & 0.4 \\
\hline & \multicolumn{5}{c}{ Correlaciones entre factores } & \\
Factor 1 & - & - & \\
Factor 2 & 0.28 & 0.1 & - & \\
Factor 3 & 0.36 &
\end{tabular}

Siguiendo el procedimiento de la prueba anterior, el análisis teórico de cada factor tampoco mostró diferencias entre ellos, por lo cual se decidió realizar un análisis factorial de segundo orden. Los resultados muestran una solución unifactorial con un valor mayor de 1.87 , que explica el $62.58 \%$ de la varianza total (Cuadro 6). 
Doris Argumedo, Karem Díaz, Arturo Calderón, Juan F. Díaz y Joseph R. Ferrari

\section{Cuadro 6}

Valores propios, porcentajes de varianza y porcentajes acumulados en el análisis factorial de segundo orden del Inventario de Procrastinación para Adultos

\begin{tabular}{cccc}
\hline Componente & Valor propio & \% de varianza & \% acumulado \\
\hline 1 & 1.88 & 62.58 & 62.58 \\
2 & 0.71 & 23.72 & 86.31 \\
3 & 0.41 & 13.69 & 100.00 \\
\hline
\end{tabular}

El análisis de componentes principales del Cuestionario de Procrastinación en la Toma de Decisiones reveló una solución unifactorial con valor propio igual 2.8 (método de Kaiser) que explica el $57.66 \%$ de la varianza total y índice Kaiser-Mayer-Olkin de 0.82 (Cuadro 7).

\section{Cuadro 7}

Análisis factorial del Cuestionario de Procrastinación en la Toma de Decisiones

\begin{tabular}{cccc}
\hline Componente & Valor propio & \% de varianza & \% acumulado \\
\hline 1 & 1.88 & 62.58 & 62.58 \\
2 & 0.71 & 23.72 & 86.31 \\
3 & 0.41 & 13.69 & 100.00 \\
Factores & Carga factorial & Comunalidad & \\
\hline Factor 1 & 0.75 & 0.87 & \\
Factor 2 & 0.51 & 0.72 & \\
Factor 3 & 0.61 & 0.78 & \\
\hline
\end{tabular}

Finalmente se realizó un análisis de correlación de Pearson para estudiar la relación entre la Escala de Procrastinación General, el Inventario de Procrastinación para Adultos y el Cuestionario de Procrastinación en la Toma de Decisiones. El Cuadro 8 presenta las correlaciones encontradas entre las escalas. Las tres escalas presentan correlaciones positivas y significativas entre sí, siendo la Escala de Procrastinación General la que presenta 
asociaciones más fuertes con las otras dos $(r=66$ y $\mathrm{r}=.56)$. El Cuestionario de Procrastinación en la Toma de Decisiones presenta la asociación más baja con relación a las otras dos pruebas ( $r=$ .49 y $r=.56$ ), probablemente debido a que mide el aspecto cognitivo en la procrastinación, a diferencia de las otras dos pruebas que apuntan a evaluar el aspecto comportamental de la procrastinación.

\section{Cuadro 8}

Correlaciones entre la Escala de Procrastinación General, el Inventario de Procrastinación para Adultos y el Cuestionario de Procrastinación en la Toma de Decisiones

\begin{tabular}{lccc}
\hline Escalas & $\begin{array}{c}\text { Inventario de } \\
\text { Procrastinación para } \\
\text { Adultos }\end{array}$ & $\begin{array}{c}\text { Escala de } \\
\text { Procrastinación } \\
\text { General }\end{array}$ & $\begin{array}{c}\text { Cuestionario } \\
\text { en la } \\
\text { Toma de Decisiones }\end{array}$ \\
\hline $\begin{array}{l}\text { Inventario de Procrastinación } \\
\text { para Adultos }\end{array}$ & - & $0.66^{* *}$ & $0.49^{* *}$ \\
\hline $\begin{array}{l}\text { Escala de Procrastinación } \\
\text { General }\end{array}$ & & - & $0.56^{* *}$ \\
\hline
\end{tabular}

Cuestionario en la

Toma de Decisiones

**La correlación es significativa al nivel 0.01 (una cola)

Dado que los resultados encontrados al conducir análisis factoriales de segundo orden para dos de las tres escalas (Escala de Procrastinación General e Inventario de Procrastinación para Adultos), mostraron una estructura unifactorial subyacente de procrastinación crónica, se procedió a analizar la composición factorial de las tres escalas de procrastinación en conjunto, esperando encontrar un sólo factor que explique el mayor porcentaje de la varianza de la procrastinación crónica. Se condujo un análisis factorial exploratorio de componentes principales con rotación Oblimin sin especificar los factores esperados, encontrándose un fac- 
Doris Argumedo, Karem Díaz, Arturo Calderón, Juan F. Díaz y Joseph R. Ferrari

tor significativo con valor propio igual a 2.2 (método de Kaiser), que explica el $71.9 \%$ de la varianza total (ver Cuadro 9).

\section{Cuadro 9}

Análisis factorial de las escalas de procrastinación

\begin{tabular}{lccc}
\hline \multicolumn{1}{c}{ Componente } & Valor propio & \% de varianza & \% acumulado \\
\hline 1 & 2.16 & 72.08 & 72.08 \\
2 & 0.53 & 17.75 & 89.82 \\
3 & 0.31 & 10.18 & 100.00 \\
\hline \multicolumn{1}{c}{ Escala } & Carga factorial & Comunalidad & \\
\hline $\begin{array}{l}\text { Inventario de Procrastinación } \\
\text { para Adultos }\end{array}$ & 0.86 & 0.74 & \\
$\begin{array}{l}\text { Escala de Procrastinación } \\
\text { General }\end{array}$ & 0.89 & 0.79 & \\
$\begin{array}{l}\text { Cuestionario de Procrastinación } \\
\text { en la Toma de Decisiones }\end{array}$ & 0.79 & 0.63 & \\
\hline
\end{tabular}

A la luz de los hallazgos, es posible concluir que las tres pruebas estudiadas miden un solo constructo en la muestra de adultos de Lima Metropolitana, es decir, la procrastinación crónica. Estos hallazgos son consistentes con los encontrados en estudios previos con muestras americanas y canadienses (Ferrari, 1992; Ferrari \& Gojkovich, 2000), así como en una muestra de estudiantes peruanos (Díaz, 2004).

\section{Procrastinación crónica según variables socio-demográficas}

A continuación se muestran los resultados referidos a la procrastinación crónica con relación a las variables sociodemográficas de la muestra. Se incluyen análisis descriptivos y análisis por contraste de hipótesis (Prueba de Diferencias de Medias de $T$ de Student y Anova One Way) para determinar dichas diferencias. Se considera además el análisis del tamaño del efecto siguiendo las 
Evaluación de la confiabilidad y la estructura factorial de tres escalas

recomendaciones de Cohen (1988) como una medida de la potencia estadística en muestras grandes.

El análisis de las puntuaciones alcanzadas por los participantes en las tres escalas de procrastinación crónica se muestra en el Cuadro 10.

Cuadro 10

Distribución de la muestra: medias y desviaciones estándar

\begin{tabular}{lcr}
\hline \multicolumn{1}{c}{ Escalas } & $M$ & \multicolumn{1}{c}{$D E$} \\
\hline Inventario de Procrastinación para Adultos & 38.56 & 9.29 \\
Escala de Procrastinación General & 51.70 & 10.34 \\
Cuestionario de Procrastinación en la Toma de Decisiones & 12.22 & 10.34 \\
\hline
\end{tabular}

$N=514$

Con respecto a las variables socio-demográficas, el sexo del participante parece no establecer diferencias en la procrastinación crónica evaluada tanto por el Inventario de Procrastinación para Adultos como por el Cuestionario de Procrastinación para la Toma de Decisiones. Centrándonos en la Escala de Procrastinación General, se observan diferencias significativas entre hombres $(M=53, D E=10.03)$ y mujeres $(M=51.12, D E=10.08), \mathrm{t}(510)$ $=2.11, \mathrm{p}<0.05$, con un tamaño de efecto nulo $(d=0.18)$, por lo tanto no podemos considerar dichas diferencias relevantes a nivel conceptual.

Con relación a la edad, observamos que existen diferencias significativas en los niveles de procrastinación evaluados por la Escala de Procrastinación General $(\mathrm{t}(510)=3.93, \mathrm{p}<0.01)$, con un tamaño de efecto pequeño $(d=0.35)$. Tenemos entonces que, si bien los adultos jóvenes $(M=53.74, D E=9.92)$ exhiben mayor tendencia a procrastinar motivados por la necesidad de experimentar altos niveles de activación al ponerse en el limite del tiempo 
en comparación con los adultos medios $(M=50.28, D E=9.98)$, estas diferencias no son importantes.

El nivel de instrucción parece establecer niveles diferenciales significativos en el nivel de procrastinación. En particular, se observa mayor tendencia a procrastinar, sea por activación (Escala de Procrastinación General), por evitación (Inventario de Procrastinación para Adultos) o por demora en las decisiones (Cuestionario de Procrastinación en la Toma de Decisiones), en la población con educación secundaria, en contraste con quienes cuentan con una educación superior técnica o universitaria. Sin embargo, estas diferencias carecen de importancia dado el tamaño de efecto pequeño $(\mathrm{d}<0.5$ ). El Cuadro 11 presenta los estadísticos de procrastinación crónica evaluada por las tres escalas según nivel de instrucción.

\section{Cuadro 11}

Procrastinación crónica según instrucción

\begin{tabular}{|c|c|c|c|c|c|c|c|c|c|c|c|c|}
\hline \multirow[t]{2}{*}{ Escalas } & \multicolumn{3}{|c|}{ Secundarıa } & \multicolumn{3}{|c|}{ Superior Técnica } & \multicolumn{3}{|c|}{ Universitaria } & \multirow[b]{2}{*}{$F$} & \multirow[b]{2}{*}{$p$} & \multirow[b]{2}{*}{ Eta } \\
\hline & $n$ & $M$ & $D E$ & $n$ & $M$ & $D E$ & $n$ & $M$ & $D E$ & & & \\
\hline $\begin{array}{l}\text { Escala de } \\
\text { Procrastinación } \\
\text { General }\end{array}$ & 304 & 53.6 & 10.2 & 102 & 49.0 & 8.9 & 106 & 50.4 & 9.9 & 9.980 & .00 & 0.38 \\
\hline $\begin{array}{l}\text { Inventario de } \\
\text { Procrastinación } \\
\text { para Adultos }\end{array}$ & 303 & 39.9 & 9.1 & 102 & 36.8 & 8.5 & 107 & 36.03 & 8.2 & 9.810 & 0.00 & 0.37 \\
\hline $\begin{array}{l}\text { Cuestionario de } \\
\text { Procrastinación en } \\
\text { la Toma de } \\
\text { Decisiones }\end{array}$ & 304 & 12.9 & 13.0 & 103 & 11.5 & 4.2 & 107 & 11.0 & 3.6 & 7.740 & 0.00 & 0.29 \\
\hline
\end{tabular}

Finalmente, el estrato socioeconómico parece establecer diferencias significativas en el nivel de procrastinación crónica cuando 
ésta se asocia a la tendencia a evitar tareas que puedan cuestionar las capacidades personales $(F=14.03, p<0.01)$. Tenemos entonces que los niveles de procrastinación por evitación son menores entre los estratos A, B y C en contraste con la población perteneciente a los estratos D y E, ello con un tamaño de efecto mediano $(E t a=$ 0.76). El Cuadro 12 muestra los descriptivos de procrastinación, niveles de significación y tamaño del efecto por estrato socio-económico para cada prueba utilizada.

Cuadro 12

Procrastinación crónica según nivel socioeconómico

\begin{tabular}{|c|c|c|c|c|c|c|c|c|c|c|c|c|c|c|c|}
\hline \multirow[t]{2}{*}{ Escalas } & \multicolumn{3}{|c|}{ Estrato A } & \multicolumn{3}{|c|}{ Estrato B } & \multicolumn{3}{|c|}{ Estrato C } & \multicolumn{3}{|c|}{ Estrato D-E } & \multirow[b]{2}{*}{$F$} & \multirow[b]{2}{*}{$p$} & \multirow[b]{2}{*}{ Eta } \\
\hline & $n$ & $M$ & $D E$ & $n$ & $M$ & $D E$ & $n$ & $M$ & $D E$ & $n$ & $M$ & $D E$ & & & \\
\hline $\begin{array}{l}\text { Escala de } \\
\text { Procrastınacıón } \\
\text { General }\end{array}$ & 20 & 50.0 & 113 & 90 & 48.0 & 97 & 144 & 51.0 & 97 & 260 & 53.7 & 10.0 & 6.42 & 0.00 & 0.37 \\
\hline $\begin{array}{l}\text { Inventario de } \\
\text { Procrastınación } \\
\text { para Adultos }\end{array}$ & 20 & 351 & 105 & 90 & 344 & 8.1 & 144 & 37.5 & 81 & 258 & 40.7 & 9.0 & 14.02 & 0.00 & 0.76 \\
\hline $\begin{array}{l}\text { Cuestionario de } \\
\text { Procrastınación } \\
\text { en la Toma de } \\
\text { Decisiones }\end{array}$ & 20 & 103 & 37 & 90 & 108 & 37 & 144 & 116 & 4.2 & 260 & 13.2 & 5.0 & 8.20 & 0.00 & 0.46 \\
\hline
\end{tabular}

$N=514$

\section{Discusión}

La literatura revisada enfatiza la necesidad de evaluar la intensidad y frecuencia de conductas de postergación en la descripción y diagnóstico de la procrastinación crónica. En ese sentido, el estudio de la validez y confiabilidad de tres instrumentos para la evaluación de la procrastinación crónica permite no sólo incorporar herramientas para su investigación sino también, a partir de 
los resultados, observar como se manifiesta el fenómeno en adultos residentes limeños.

La Escala de Procrastinación General presenta un alto índice de consistencia interna $(\alpha=0.81)$, resultando similar a lo encontrado en investigaciones realizadas con estudiantes universitarios y adultos en Estados Unidos (Ferrari 1992b), Canadá (Lay, 1986; Lay \& Burns, 1991), Holanda (Lay \& Schouwenburg, 1993), Australia e Inglaterra (Ferrari, O'Callahan \& Newbegin, 2004). El análisis discriminativo de los ítemes de la escala revela sin embargo que dos de los veinte ítemes ( 5 y 11) presentan poca capacidad de discriminación. El ítem 5 parece recoger manifestaciones socioeconómicas que no se encuentran de forma tan evidente en los otros ítemes. Este ítem "usualmente respondo $e$-mails varios días después de haberlos recibido" requiere que el participante utilice $y / o$ esté familiarizado con el uso de Internet $y$, dadas las características de la muestra, es posible que una buena parte de los entrevistados, aunque conozca de su existencia, no tenga acceso a Internet por su condición económica. Con respecto al ítem 11 "cuando me preparo para salir, rara vez tengo que hacer algo a último minuto", es importante señalar en primer lugar que este ítem ha mostrado un comportamiento atípico al ser evaluado en una muestra de estudiantes universitarios limeños (Díaz, 2004). Como se analizó en aquella ocasión, es posible que la poca capacidad de discriminación mostrada se deba a la forma en que se ha enunciado, es decir, mencionar la realización de dos acciones casi al mismo tiempo no permite comprender su verdadera intención: "no hay tareas pendientes, importantes o urgentes para cuando es momento de salir de casa". Asimismo, la inclusión del adverbio de frecuencia "rara vez" se presenta redundante y contradictorio al tener que contrastarse con alternativas de respuesta que ya incluyen frecuencias en el hábito que se evalúa. Es necesario, entonces, cambiar el enunciado del ítem cuidando de mantener su sentido teórico así como su claridad. Se deja a futuras investigaciones 
Evaluación de la confiabilidad y la estructura factorial de tres escalas

estudiar enunciados alternativos más claros y precisos para su cambio.

El análisis factorial efectuado mostró que, si bien inicialmente los ítemes se agrupan en tres factores, éstos no muestran diferencias teóricas sustanciales entre sí que permitan establecer áreas temáticas de la procrastinación, ya sea en tareas cotidianas, de mantenimiento personal, en las relacionadas al trabajo, etc. Asimismo, la teoría de procrastinación no ha señalado la existencia de categorías o características internas que conformen el constructo, así como tampoco éstas se han establecido para la escala evaluada. Al respecto Schouwenburg (Comunicación Personal, 15 de Noviembre, 2003) señala haber encontrado, en muestras de adultos y estudiantes holandeses, repetidamente cinco factores al interior de esta escala, distintos a los hallados en la muestra de adultos peruanos. En contraste, Pychyl (Comunicación Personal, 4 de Setiembre, 2004) señala que la escala muestra ser unifactorial en las investigaciones realizadas en muestras de estudiantes y adultos canadienses.

La distribución de los ítemes en los factores parece obedecer a asociaciones características que se realizan en cada cultura con respecto a las tareas evaluadas, mas no establecen áreas diferenciadas que conformen diversas dimensiones de la procrastinación crónica. Ello permite entender, en parte, la gran variación de las agrupaciones de ítemes establecidas al interior de la escala en las tres poblaciones mencionadas. La conducción de un análisis factorial de segundo orden con los factores hallados ha permitido encontrar un factor de procrastinación general subyacente a las agrupaciones de ítemes. Se deduce entonces que las áreas en las que los ítemes se agrupan responden a la variable de procrastinación crónica en conjunto y que estas agrupaciones incrementan la fuerza predictiva de la conducta procrastinadora por la escala. 
El Inventario de Procrastinación para Adultos alcanzó igualmente un alto nivel de consistencia interna $(\alpha=0.81)$, similar a los índices reportados en investigaciones anteriores con poblaciones canadienses, americanas, inglesas y australianas (Ferrari et al., 1995; Ferrari et al., 2004). El análisis de los ítemes que componen el inventario mostró la poca discriminación del ítem 6 "si se dictase un curso sobre como hacer las cosas a tiempo, yo iría", el cual describe un uso muy aceptado y promovido en otros contextos culturales, donde grupos de autoayuda y talleres para determinados problemas son populares entre la población. Es posible que, si bien entre los limeños participantes se esperaría que un tema como la postergación y posibles soluciones a través de un taller sea una actividad atractiva, esta practica es poco común en el contexto peruano. Se entiende que la falta de familiaridad con la actividad medie en la evaluación del ítem y no se relacione directamente con la conducta que se desea evaluar. Sería recomendable buscar una actividad alternativa para modificar este ítem, que permita una mayor identificación del problema de procrastinación y que sea común a la mayor parte de la población peruana.

Respecto a la solución factorial hallada, se encontró nuevamente que los ítemes del Inventario de Procrastinación para Adultos, aunque se agrupan en tres factores, muestran poca diferencia teórica entre sí. El análisis factorial de segundo orden verifica la procrastinación subyacente que el inventario evalúa. Se encuentra necesario que futuras investigaciones repliquen este estudio con el fin de verificar la configuración de áreas determinadas por estilos de vida característicos de nuestra sociedad, establecer cuáles son y estudiar el comportamiento de los ítemes analizados para ver su desempeño y pertinencia en la escala.

El Cuestionario de Procrastinación en la Toma de Decisiones muestra un índice de confiabilidad adecuado $(\alpha=0.84)$ y similar a lo observado en investigaciones previas en poblaciones americanas, 
australianas e inglesas (Ferrari et al., 1995, 2004; Mann et al., 1997). Además, se encontró que la prueba es unidimensional, lo cual corresponde a los reportes sobre las propiedades psicométricas de esta escala. La manifestación cognitiva de la procrastinación crónica al parecer no se ve influida y alterada por variaciones culturales o la concepción que se tenga de la tarea; es entonces posible que los procesos cognitivos sean independientes y compartidos por todos.

Con respecto a la validez de constructo de las tres escalas de procrastinación crónica, las correlaciones fuertes y significativas encontradas para las escalas coinciden con los hallazgos de otras investigaciones donde, en conjunto, se utilizan las tres pruebas para evaluar la procrastinación crónica en general y las posibles motivaciones subyacentes a este fenómeno (Ferrari, 1992b; Ferrari \& Gojkovich, 2000).

Con respecto a las variaciones en el nivel de procrastinación según diversas variables socio-demográficas, en concordancia con otras investigaciones (Harriott \& Ferrari, 1996) no se han encontrado diferencias que tengan una valor conceptual relevante según sexo, edad y nivel instrucción. Los análisis de la variable socioeconómica arrojaron que existen diferencias significativas y con efecto moderado en la procrastinación motivada por la evitación de tareas pesadas y/o de amenazas a la autoestima entre los estratos socioeconómicas A, B y C, en contraste con el sector D y $\mathrm{E}$, siendo estos últimos los que presentan mayores niveles de procrastinación motivada por la evitación. Es posible que la falta de recursos y formación, así como un patrón de personalidad específico, contribuyan a fomentar la cronicidad de la procrastinación en este sector poblacional. Este hallazgo no puede ser contrastado con lo encontrado en países de habla inglesa donde la población resulta ser más homogénea cultural y económicamente. Las diferencias socioculturales en países latinoamericanos con carencias 
económicas fuertes parecen influir en las preferencias, modos de enfrentar y de vivir de sus habitantes. Sin embargo, se hace necesaria una evaluación muestral más grande para poder generalizar los resultados, así como una comparación con poblaciones de otras ciudades del interior del país que ayuden a comprender el rol de la variable socioeconómica y cultural en toda su dimensión en la presentación de la procrastinación crónica.

Para finalizar y con relación al estudio en su totalidad, podemos concluir que las escalas utilizadas para la evaluación de la procrastinación crónica se presentan validas y confiables para la población adulta limeña estudiada. Sin embargo, se plantea la necesidad de estudiar y modificar algunos ítemes que parecen estar afectados por variables socioeconómicas y culturales particulares de nuestro contexto. Estos hallazgos concuerdan con los encontrados en una población universitaria de Lima (Díaz, 2004). Por otro lado, es importante resaltar la pertinencia del uso de las tres escalas en la evaluación global de procrastinación crónica pues, al parecer, permiten en conjunto la medición de los aspectos comportamentales y cognitivos de la procrastinación. Surge como propuesta la elaboración de una sola escala que integre ambos aspectos y las motivaciones que a la base elicitan este fenómeno, compuesta además por reactivos que prueben su valor transcultural.

\section{Referencias}

Apoyo, Opinión y Mercado. (2002). Niveles socioeconómicos de la gran Lima. Lima: Apoyo.

Cohen, J. (1988). Statistical power analysis for the behavioral sciences. Hillsdale, NJ: Lawrence Erlbaum.

Díaz, K. (2004). Procrastinación crónica y estilos de personalidad en una muestra de estudiantes universitarios de Lima 
Evaluación de la confiabilidad y la estructura factorial de tres escalas

Metropolitana. Tesis de licenciatura no publicada, Pontificia Universidad Católica del Perú, Lima.

Ellis, A. \& Knaus, W. (1977). Overcoming procrastination. Nueva York: Penguin Putnam.

Ferrari, J. (1992a). Procrastination in the workplace: Attributions for failure among individuals with similar behavioral tendencies. Personality and Individual Differences, 13, 315-319.

Ferrari, J. (1992b). Psychometric validation of two procrastination inventories for adults: Arousal and avoidance measures. Journal of Psychopathology and Behavioral Assessment, 14, 97-110.

Ferrari, J. (2001). Procrastination as self-regulation failure of performance: Effects of cognitive load, self-awareness and time limits on "working best under pressure". European Journal of Personality, 15, 391-406.

Ferrari, J. \& Dovidio, J. (2000). Examining behavioral processes in indecision: Decisional procrastination and decision-making style. Journal of Research in Personality, 34, 127-137.

Ferrari, J. \& Gojkovich, P. (2000). Procrastination and attention: Factor analysis of attention deficit, boredomness, intelligence, self-esteem, and task delay frequencies. Journal of Social Behavior and Personality, 15, 185-197.

Ferrari, J., Johnson, J. \& McCown, W. (1995). Procrastination and task avoidance. Nueva York: Plenum Press.

Ferrari, J., O’Callahan, J. \& Newbegin, I. (2004). Prevalence of procrastination in the United States, United Kingdom, and Australia: Arousal and avoidance delays among adults. North American Journal of Psychology, 6, 1-6.

Harriott, J. \& Ferrari, J. (1996). Prevalence of procrastination among samples of adults. Psychological Reports, 78, 611616.

Hernández, R., Fernández, C. \& Baptista, P. (2000). Metodología de la investigación (2da. ed.). México: McGraw-Hill.

Lay, C. (1986). At last, my research article on procrastination. Journal of Research in Personality, 20, 474-495. 
Lay, C. \& Burns, P. (1991). Intentions and behavior in studying for an examination: The role of trait procrastination and its interaction with optimism. Journal of Social Behavior \& Personality, 6, 605-617.

Lay, C. \& Schouwenburg, H. C. (1993). Trait procrastination, time management, and academic behavior. Journal of Social Behavior and Personality, 8, 647-662.

Mann, L. (1982). Decision-Making Questionnaire. Manuscrito no publicado, Flinders University of South Australia.

Mann, L., Burnett, P., Radford, M. \& Ford, S. (1997). The Melbourne Decision Making Questionnaire: An instrument for measuring patterns for coping with decisional conflicts. Journal of Behavioral Decision Making, 10(1), 1-19.

Merriam-Webster. (2004). Merriam-Webster's New Collegiate Dictionary (11va. ed.). Springfield, MA: Merriam-Webster.

Real Academia Española. (2002). Diccionario de la lengua española (22da. ed., Vol. 2). Madrid: Espasa Calpe.

Schouwenburg, H., Lay, C., Pychyl, T. \& Ferrari, J. (2004). Counseling the procrastinator in academic settings. Washington, DC: American Psychological Association.

Steel, P. (2003). The nature of procrastination. Manuscrito no publicado, Universidad de Calgary, Alberta, Canadá. 